\title{
Plasma Glial Fibrillary Acidic Protein and Neurofilament-Light for the Diagnostic and Prognostic Evaluation of Frontotemporal Dementia
}

\author{
Nuole Zhu \\ Hospital de la Santa Creu i Sant Pau https://orcid.org/0000-0002-0015-9455 \\ Miguel Santos-Santos \\ Hospital de la Santa Creu i Sant Pau \\ Ignacio Illán-Gala \\ Hospital de la Santa Creu i Sant Pau \\ Victor Montal \\ Hospital de la Santa Creu i Sant Pau Institut de Recerca \\ Teresa Estellés \\ Hospital de la Santa Creu i Sant Pau \\ Isabel Barroeta \\ Hospital de la Santa Creu i Sant Pau \\ Miren Altuna \\ Hospital de la Santa Creu i Sant Pau \\ Javier Arranz \\ Hospital de la Santa Creu i Sant Pau \\ Laia Muñoz \\ Hospital de la Santa Creu i Sant Pau Institut de Recerca \\ Olivia Belbin \\ Hospital de la Santa Creu i Sant Pau Institut de Recerca \\ Isabel Sala \\ Hospital de la Santa Creu i Sant Pau \\ Maria Belén Sanchez-Saudinos \\ Hospital de la Santa Creu i Sant Pau Institut de Recerca \\ Andrea Subirana \\ Hospital de la Santa Creu i Sant Pau Institut de Recerca \\ Laura Videla \\ Hospital de la Santa Creu i Sant Pau Institut de Recerca \\ Rafael Blesa \\ Hospital de la Santa Creu i Sant Pau \\ Jordi Clarimón \\ Hospital de la Santa Creu i Sant Pau Institut de Recerca \\ Maria Carmona-Iragui \\ Hospital de la Santa Creu i Sant Pau \\ Juan Fortea \\ Hospital de la Santa Creu i Sant Pau \\ Alberto Lleó \\ Hospital de la Santa Creu i Sant Pau \\ Daniel Alcolea ( DAlcolea@santpau.cat) \\ https://orcid.org/0000-0002-3819-3245
}

Research

Keywords: GFAP, Neurofilament, Frontotemporal dementia, Plasma biomarkers

Posted Date: October 11th, 2021

DOI: https://doi.org/10.21203/rs.3.rs-958956/v1 


\section{Abstract}

Background: Astrocytes play an essential role in neuroinflammation and are involved in the pathogenesis of neurodenegerative diseases. The Study of Glial fibrillary acidic protein (GFAP), an astrocytic damage marker, may be helpful to better understand the different neurodegenerative diseases. We investigated the diagnostic performance of plasma GFAP (pGFAP), plasma neurofilament ( $\mathrm{pNfL}$ ) and their combination in frontotemporal dementia (FTD) and Alzheimer's disease (AD). We studied their clinical utility in predicting disease progression.

Methods: We measured pGFAP and pNfL concentrations in 72 FTD, 56 AD and 83 cognitively normal participants (CN) using Single Molecule Array technology. Of 211 participants, 199 had CSF and 122 had MRI. We compared cross-sectional biomarker levels between groups, studied their diagnostic performance and assessed correlation with CSF biomarkers, cognitive performance and cortical thickness. The prognostic performance was investigated analyzing cognitive decline between group comparisons by tertile.

Results: Unlike pNfL, which was increased similarly in both clinical groups, pGFAP was increased in FTD but lower than in AD (all p<0.01). The combination of both plasma markers improved the diagnostic performance to discriminate FTD from AD (combination AUC 0.78; pGFAP AUC 0.7; pNfL AUC 0.61, all p $<0.05$ ). In FTD, pGFAP correlated with cognition, CSF and plasma NfL, and cortical thickness (all $p<0.05$ ). The higher tertile of pGFAP was associated with greater change in MMSE score and poor cognitive outcome during follow-up both in FTD (1.40 points annually, HR 3.82, $\mathrm{p}<0.005)$ and $\mathrm{AD}(1.20$ points annually, HR $2.26, \mathrm{p}<0.005)$.

Conclusions: pGFAP and pNfL differed in FTD and AD, their combination could be useful to distinguish the two diseases. pGFAP could also be used to track disease severity and predict greater cognitive decline during follow-up in patients with FTD.

\section{Background}

Frontotemporal dementia (FTD) is a progressive neurodegenerative condition characterized by clinical, genetic and neuropathologic heterogeneity. The clinical manifestation of FTD may overlap with psychiatric or other neurodegenerative disorders, such as Alzheimer's disease (AD). Diagnosis is thus a clinical challenge.

In the past two decades, many efforts have been made to find imaging or fluid biomarkers for FTD ${ }^{1-6}$. In spite of the notable advances, we still do not have pathophysiological markers of FTD to be used in clinical practice. Recently, some studies have shown that concentrations of NfL, a marker of axonal damage, are increased in CSF and blood in FTD patients. Moreover, their levels reflect disease severity and predict clinical progression ${ }^{1-6}$. However, NfL is not specific of FTD and can be increased in other neurodegenerative diseases, such as AD or Lewy body dementia. There is considerable overlap in NfL levels between the different conditions ${ }^{1-6}$.

Neuroinflammation and neurodegeneration are highly interrelated processes in neurological diseases ${ }^{7}$. Increased levels of Glial Fibrillary acidic protein (GFAP) in CSF and plasma, a marker of astrogliosis, have been described in different neurodegenerative diseases ${ }^{8-17}$. In AD, recent studies also showed that plasma GFAP (pGFAP) levels are associated with amyloid pathology. Furthermore, some studies have suggested that GFAP could be a marker of disease severity ${ }^{8-21}$ and be a prognostic marker for progression to AD dementia in cognitively normal older adults ${ }^{15-21}$. Likewise, other studies reported increased CSF and pGFAP concentrations in FTD, even though the results are not consistent across studies ${ }^{10,12,13,22-26}$. Although previous studies did not detect blood GFAP level changes in FTD ${ }^{10,12,13}$, some studies observed elevated pGFAP in all different FTD subgroups ${ }^{23-26}$. Higher pGFAP concentrations have been associated with greater functional impairment and disease severity in two studies ${ }^{13,24}$, although the role of pGFAP in predicting disease progression in FTD and its potential use for diagnosis alone or in combination with other biomarkers remains unclear.

In this study, our primary research aim was to determine the potential use of pGFAP, pNfL or their combination to distinguish patients with FTD from $A D$ and from cognitively normal participants (CN), and to study their association with disease progression in FTD. As a secondary objective we aimed to study the correlation of pGFAP with cognition, other biomarkers and structural measures in neuroimaging.

\section{Methods}

Study participants and classification. We collected clinical and biomarker information of 211 participants with available plasma samples from the Sant Pau Initiative on Neurodegeneration (SPIN) cohort, a multimodal biomarker platform for the study of neurodegenerative diseases ${ }^{27}$. Participants were classified into one of the following clinical groups according to internationally accepted diagnostic criteria: 72 patients with probable FTD-related clinical syndromes and negative AD biomarkers: 33 patients with behavioral variant of frontotemporal dementia (bvFTD) ${ }^{28}, 7$ semantic variant of primary progressive aphasia (svPPA) ${ }^{29}, 14$ nonfluent variant of primary progressive aphasia (nfvPPA) ${ }^{29}$ and 18 progressive supranuclear palsy-corticobasal syndrome spectrum (PSP-CBD) ${ }^{30,31}$. We also included 56 patients with Alzheimer disease (AD) with evidence of the $A D$ pathophysiological process ${ }^{32,33}$ either through CSF biomarkers $(n=51)$ or amyloid-PET $(n=5)$ and 83 CN that had normal CSF values of $A \beta_{42} / A \beta_{40}$, pTau181 and neuropsychological evaluation within normal range ${ }^{27}$. 
Plasma availability was the prerequisite to include the participants in the study. CSF samples were available in 199 participants (94\%). A subset of 122 participants (58\%) had undergone 3 Tesla structural brain MRI. APOE genotype was available in 203 participants (96\%). 202 participants (96\%) were longitudinally followed up and underwent a comprehensive evaluation. All participants had a Mini-mental state examination (MMSE) and a Global Deterioration Scale of Reisberg (GDS) score at the time of diagnosis, and repeated measures of MMSE were obtained during the follow-up. In a subset of 45 FTD patients, Frontotemporal Dementia Rating Scale (FTD-FRS) was available at the time of diagnosis.

Blood and CSF sample analysis. Blood was collected in 10ml EDTA tubes and immediately transferred to our laboratory where they were centrifuged and aliquoted within 2 hours after extraction. CSF samples were collected on the same day of blood extraction and processed in polypropylene tubes following international recommendations ${ }^{34}$. All samples were processed and aliquoted within the first two hours after the lumbar puncture. Plasma and CSF aliquots were stored at $-80^{\circ} \mathrm{C}$ until analysis. pGFAP and $\mathrm{pNfL}$ concentrations were measured using SR-X single molecule array (SIMOA). CSF AD core biomarkers (AB42, A 40 , tTau and pTau181) were measured in the fully-automated platform Lumipulse (Fujirebio-Europe), and levels of CSF NfL (Uman Diagnostics) and CSF YKL-40 (MicroVue ${ }^{\mathrm{TM}}$, Quidel) were measured through ELISA using previously reported methods ${ }^{32,33}$. The preanalytical protocol for blood and CSF in the SPIN cohort has been described in detail previously 27.

Statistical analysis. Chi-squared's test was used to compare sex and APOE status frequencies between groups. Continuous variables were expressed as means and standard deviation (SD). Distributions for demographic and biomarker data were assessed using Shapiro-Wilk's test and homogeneity of variances was checked by Levene's test. Biomarker raw values not following a normal distribution were log-transformed to achieve a normal distribution. Age- and sex-adjusted analysis of covariance (ANCOVA) followed by post-hoc Tukey test was used to compare pGFAP and pNfL levels between groups. To investigate the potential use of pGFAP, pNfL or their combination to distinguish patients with FTD from AD and CN, we assessed areas under the curve (AUC) by receiver operating characteristic (ROC) analyses. ROC curves were compared using DeLong's test. To study the association of pGFAP with disease progression, we divided participants in 3 tertile groups according to their pGFAP levels. A linear-mixed model was used to assess the association of pGFAP with cognitive decline during the follow-up. We included age, sex, MMSE score at the time of diagnosis, pGFAP tertile and its interaction with time as fixed factors, and modeled random intercepts and slopes at the participant level to account for repeated measures. Multivariate Cox regression analysis adjusting for sex and baseline age and Kaplan Meier curves were performed to analyze the predictive value of pGFAP for significant cognitive impairment (MMSE score <20). In order to investigate the relationship of pGFAP concentrations with demographics, cognitive scores, CSF biomarkers and pNfL concentrations, we assessed Spearman's correlations. Cortical thickness was computed with Freesurfer software ${ }^{35}$ (version 5.1) (https://surfer.nmr.mgh.harvard.edu/fswiki), and its correlation with pGFAP was assessed through general linear model, including age and sex as fixed factors.

Statistical significance for all tests was set at $5 \%(\mathrm{p}=0.05)$ and corrected for multiple comparisons. All the statistical analyses were performed using packages "psych" (v. 2.0.8), "ggplot2" (v. 3.3.3), "pROC" (v. 1.16.2), "ImerTest” (v. 3.1-3), "nlme" (v. 3.1-148), "multcomp" (v. 1.4-13), "survival” (v. 3.211 ) and "survminer" (v. 0.4.9) as implemented in R statistical software version R 4.0.2 (http://www.R-project.org).

\section{Results}

\section{Demographics and clinical data}

Table 1 shows demographics and clinical data, CSF and plasma biomarker levels in the FTD, AD and CN groups. Controls were significantly younger than symptomatic patients, but there was no difference in age between the symptomatic groups. Controls had more years of education than the symptomatic groups, and there were no differences in sex between the groups. APOEE 4 genotype was more frequent in the AD group. Both baseline and last MMSE scores were lower in disease groups compared with controls. Within FTD subgroups, baseline and last MMSE scores were lower in svPPA compared to other subgroups. The mean follow-up period was $3.4( \pm 2.3)$ years in FTD, $3.9( \pm 2)$ in $A D$ and $4( \pm 1.7)$ in $C N$. Follow-up period did not differ between the groups.

Age correlated with pGFAP (Rho 0.35, p<0.001) concentrations in the control group, but not with pNfL levels. No difference in pGFAP concentration was found between male and female participants. $p N f L$ levels were higher in male in the whole sample $(p=0.05)$, and in the $A D$ group ( $p=0.04)$, but not in $\mathrm{CN}$ or in the FTD groups.

TABLE 1. Demographics and clinical characteristics, CSF and plasma biomarkers concentrations in CN, FTD and AD 


\begin{tabular}{|c|c|c|c|c|c|c|c|}
\hline & \multirow[t]{2}{*}{$\mathrm{CN}$} & \multicolumn{5}{|l|}{ FTD } & \multirow[t]{2}{*}{$A D$} \\
\hline & & bvFTD & nfvPPA & svPPA & PSP-CBD & All FTD & \\
\hline $\mathbf{N}$ & 83 & 33 & 14 & 7 & 18 & 72 & 56 \\
\hline Age, years & $58(8.5)^{\mathrm{b}, \mathrm{c}}$ & $68.3(10.6)^{a}$ & $72.8(5.6)^{a}$ & $73.1(11.1)^{\mathrm{a}}$ & $72.9(5.4)^{\mathrm{a}}$ & $70.8(8.9)^{a}$ & $70.8(7.2)^{\mathrm{a}}$ \\
\hline \multicolumn{8}{|l|}{ Sex } \\
\hline Female & 49 & 10 & 9 & 4 & 11 & 34 & 31 \\
\hline Male & 34 & 23 & 5 & 3 & 7 & 38 & 25 \\
\hline \multicolumn{8}{|l|}{$\begin{array}{l}\text { APOE } \varepsilon 4 \\
\text { status }\end{array}$} \\
\hline$\varepsilon 4+$ & $23^{c}$ & $3^{c}$ & $3^{c}$ & $2^{\mathrm{c}}$ & $2^{c}$ & $10^{c}$ & $27^{a, b, d-g}$ \\
\hline$\varepsilon 4-$ & $57^{c}$ & $29^{c}$ & $11^{\mathrm{c}}$ & $5^{c}$ & $14^{c}$ & $59^{c}$ & $27^{a, b, d-g}$ \\
\hline $\begin{array}{l}\text { Follow up } \\
\text { time, } \\
\text { years }\end{array}$ & $3.9(1.7)$ & $4.1(2.5)$ & $3.1(2.3)$ & $4.8(1.7)$ & $1.9(1)$ & $3.4(2.3)$ & $4(2)$ \\
\hline $\begin{array}{l}\text { Education, } \\
\text { years }\end{array}$ & $15.4(4.1)^{\mathrm{b}-\mathrm{g}}$ & $12.9(5.6)^{\mathrm{a}}$ & $13.3(4.9)^{\mathrm{a}}$ & $14.8(5.2)^{\mathrm{a}}$ & $11.3(3.7)^{\mathrm{a}}$ & $12.7(5)^{a}$ & $10.4(4.7)^{\mathrm{a}}$ \\
\hline $\begin{array}{l}\text { Baseline } \\
\text { MMSE } \\
\text { score }\end{array}$ & $29.2(1)^{b-g}$ & $25.1(4.2)^{\mathrm{a}, \mathrm{f}}$ & $24.1(6.8)^{a, f}$ & 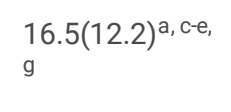 & $22.6(6.3)^{a, f}$ & $23.5(6.5)^{\mathrm{a}}$ & $23.7(3.8)^{\mathrm{a}}$ \\
\hline $\begin{array}{l}\text { Last } \\
\text { MMSE } \\
\text { score }\end{array}$ & $29.3(1)^{\mathrm{b}-\mathrm{g}}$ & $16.6(10)^{a . F}$ & $14(12.2)^{a, f}$ & $0.4(0.9)^{a, c-e, g}$ & $11.6(12.4)^{\mathrm{a}}$ & $13.3(11.4)^{\mathrm{a}}$ & $15.5(9.5)^{\mathrm{a}}$ \\
\hline $\begin{array}{l}\text { pGFAP, } \\
\mathrm{pg} / \mathrm{ml}\end{array}$ & $\begin{array}{l}134.3(45.4)^{b-} \\
d, f-g\end{array}$ & $193.1(100.1)^{\mathrm{a}}$ & $224.6(77.2)$ & $159.3(279.1)^{\mathrm{a}}$ & $271.2(148.2)^{a}$ & $234.9(141.9)^{a, b}$ & $319.8(135.1)^{\mathrm{a}, \mathrm{c}}$ \\
\hline $\begin{array}{l}\mathrm{pNfL}, \\
\mathrm{pg} / \mathrm{ml}\end{array}$ & $18(20.2)^{b-e, g}$ & $41.9(60.1)^{a}$ & $34.9(17.2)^{a}$ & $31.3(14.5)$ & $34.3(18.1)^{a}$ & $37.6(42.3)^{a}$ & $26.5(13.3)^{\mathrm{a}}$ \\
\hline $\begin{array}{l}\text { CSF NfL, } \\
\mathrm{pg} / \mathrm{ml}\end{array}$ & $\begin{array}{l}494.8(274.3)^{b} \\
c, f, g\end{array}$ & $1436.3(930.6)^{a}$ & 1944.5(1146.7) & $2394.6(637.8)^{a}$ & $1739.3(1607.3)^{a}$ & $\begin{array}{l}1654.1(1187.6)^{a}, \\
c\end{array}$ & $\begin{array}{l}1286.4(1136.9)^{a}, \\
b, f\end{array}$ \\
\hline $\begin{array}{l}\text { CSF YKL- } \\
40, \mathrm{pg} / \mathrm{ml}\end{array}$ & $190.3(49.4)^{\mathrm{b}, \mathrm{c}}$ & $265.8(64.7)^{\mathrm{a}}$ & $274.84(61)^{\mathrm{a}}$ & $311.8(37.1)^{\mathrm{a}}$ & $262.3(79.3)$ & $268.2(63.7)^{\mathrm{a}}$ & $287.3(68.1)^{a}$ \\
\hline \multicolumn{8}{|c|}{ Unless otherwise specified, values are expressed as mean (standard deviation). } \\
\hline \multicolumn{8}{|c|}{$\begin{array}{l}\text { Abbreviations: MMSE = Mini-Mental State Examination; pGFAP = plasma Glial fibrillary acidic protein; pNfL = plasma Neurofilament; } C N= \\
\text { cognitivelly normal participants; } \mathrm{FTD}=\text { Frontotemporal dementia; } \mathrm{AD}=\text { Alzheimer Disease; bvFTD = behavioral variant of frontotemporal } \\
\text { dementia; fryPPA = nonfluent variant of primary progressive aphasia; sVPPA = semantic variante of primary progressive aphasia; PSPCBD = } \\
\text { progressive supranuclear palsy-corticobasal syndrome spectrum }\end{array}$} \\
\hline
\end{tabular}

\section{Plasma GFAP and NfL concentrations differ in FTD and AD}

As shown in Figure 1, after adjusting by age and sex, pGFAP and pNfL concentration differed between FTD and AD patients.

Concentrations of pGFAP were higher in patients with FTD $(234.9 \pm 141.9 \mathrm{pg} / \mathrm{ml})$ than in $\mathrm{CN}(134.3 \pm 45.4 \mathrm{pg} / \mathrm{ml}, \mathrm{p}=0.0008)$ but lower than in $A D$ $(319.8 \pm 135.1 \mathrm{pg} / \mathrm{ml}, \mathrm{p}<0.001)$. Within FTD subgroups, pGFAP concentrations were significantly higher in SVPPA and PSP-CBD compared with controls $(p=0.03)$, and concentrations in bvFTD were lower than in AD ( $p<0.001)$ (Figure $1 A$ and $B)$.

As expected, pNfL concentrations were higher both in FTD $(37.6 \pm 42.3 \mathrm{pg} / \mathrm{ml}, \mathrm{p}<0.001)$ and AD $(26.5 \pm 13.3 \mathrm{pg} / \mathrm{ml}, \mathrm{p}=0.004)$ groups compared to CN $(18 \pm 20.2 \mathrm{pg} / \mathrm{ml})$. pNfL concentrations were increased in all FTD subgroups $(\mathrm{p}<0.05)$ compared to $\mathrm{CN}$, except svPPA, which showed a similar trends than the rest of the FTD subgroups but did not reach significance (Figure 1C and D).

\section{Diagnostic accuracy of plasma biomarkers and their combination to differentiate between FTD, AD and CN}

We next explored the diagnostic performance of pGFAP, pNfL and their combination using logistic regression to discriminate across the different groups. The basic model included age, sex and $A P O E \varepsilon 4$ allele status. We compared the diagnostic performance of this basic model with a panel including the variables in the basic model together with pGFAP and pNfL. 
A ROC curve analysis is shown in Figure 2. The combination of pGFAP and pNfL showed higher accuracy than the two plasma markers separately to differentiate FTD from AD (combination: AUC 0.78 [95\%Cl 0.70-0.86]; pGFAP: AUC 0.7 [95\%Cl 0.61-0.71]; pNfL: AUC 0.61 [95\%Cl 0.51-0.71], p=0.04 and $p=0.002$, respectively) and for the discrimination between FTD and CN (Combination: AUC 0.82 [CI95\% 0.75-0.89]; pGFAP: AUC 0.76 [95\%CI 0.680.84]; pNfL: AUC 0.81 [95\% CI 0.74-0.88], $p=0.02$ and $p=0.51$, respectively). None of the two plasma markers, individually or in combination, showed higher accuracy than the basic model. However, the addition of pGFAP and pNfL to the basic model (panel model) showed higher diagnostic performance that outperformed the basic model both in differentiating FTD from AD (AUC 0.83 [95\% Cl 0.75-0.9] vs AUC 0.69 [95\%Cl 0.6-0.79], $p=0.003$ ) and AD from CN (AUC 0.96 [95\% Cl 0.93-1] vs AUC 0.89 [95\% Cl 0.83-0.95], p=0.0007). To differentiate FTD from CN, the panel showed highest AUC compared to other methods (all p<0.05), but not compared to the basic model (AUC 0.88 [95\% $\mathrm{Cl} 0.83-0.94]$ vs $\mathrm{AUC} 0.87$ [95\% Cl 0.81 0.93], $p=0.08)$.

\section{Plasma GFAP correlated with other fluid biomarkers, cognitive and functional scores and neuroimaging}

In the whole sample, pGFAP concentrations correlated with pNfL (rho 0.53, p<0.001), CSF NfL (rho 0.52, p<0.001) and CSF YKL-40 concentrations (rho $0.49, p<0.001$ ) after adjusting by age and sex. To avoid group effects in the correlation assessment, we studied correlations of these biomarkers within groups. pGFAP concentrations correlated with plasma and CSF NfL in FTD (rho 0.49, p<0.001; rho 0.32, p=0.02) and in CN (rho 0.4, $p<0.001$; rho $0.3, p=0.016)$, but not with CSF YKL-40. By contrast, in the AD group, pGFAP correlated with pNfL only (rho $0.35, p=0.007)$ (Figure $3 A-C)$.

After adjusting by age and sex, pGFAP was also significantly associated with baseline MMSE scores in the whole sample (rho -0.58, p<0.001) and in the FTD group (Rho -0.33, p=0.005), but not in AD or in the CN groups (Figure 3D). In the FTD group, after adjusting by age, higher pGFAP concentrations were associated with lower FTD-FRS scores ( $r=-0.28, p=0.046$, Figure 3E).

We studied the correlation between pGFAP and cortical thickness in a subset of 122 participants (29 FTD, 25 AD and 68 CN) with structural MRI suitable for quantitative analyses. After adjusting by age, sex and neuroimaging acquisition center, a vertex-wise regression analysis showed a significant association between pGFAP and cortical thickness in the orbitofrontal and occipital pole regions (Figure 3F, p<0.05) in the FTD group. In the $\mathrm{AD}$ and the $\mathrm{CN}$ groups, no correlation between pGFAP concentration and cortical thickness was found (not shown).

\section{Baseline plasma GFAP levels predict cognitive decline}

To investigate the prognostic performance of pGFAP levels, we divided pGFAP levels in tertiles within each symptomatic group: low (<154pg/ml in FTD and $<228 \mathrm{pg} / \mathrm{ml}$ in AD), medium (154-240pg/ml in FTD and 228-366pg/ml in AD) and high (>240pg/ml in FTD and >366pg/ml in AD).

A linear-mixed model analysis was used to assess the relationship between baseline pGFAP concentration and cognitive decline measured by change in MMSE score during follow-up. After adjusting by age, sex and baseline MMSE score, patients in the highest pGFAP tertile showed a greater change in MMSE compared to those in the lowest tertile in both FTD (mean loss of 1.40 points annually, $p=0.003$ ) and in AD (mean loss of 1.20 points annually, $\mathrm{p}<0.001$ ) (Figure $4 \mathrm{~A}$ and $\mathrm{B}$ ).

Kaplan Meier curves (Figure 5) and Cox regression analyses including baseline age and sex as covariates were used to assess progression to moderate cognitive impairment (MMSE score < 20). Compared to the lowest tertile, the highest tertile of pGFAP was associated with increased risk of poor cognitive outcome both in FTD and in AD (1.40 points annually, HR 3.82 in FTD; 1.20 points annually, HR 2.26 in AD, both p<0.001).

\section{Discussion}

In this study, we found that pGFAP is increased in FTD, and that its concentration differs from that in AD patients. pGFAP also correlated with neuronal damage biomarkers, cognitive and functional scores and with structural imaging measures in FTD. Importantly, a higher pGFAP concentration was associated with a greater change in MMSE score during follow-up and predicted progression to moderate cognitive impairment in FTD. Our findings suggest that pGFAP could be a useful marker of disease severity and prognostic assessment in FTD, in addition to its role in AD.

Emerging evidence suggests that astrocytes play an essential role in neuroinflammation and are involved in the pathophysiology of several neurological diseases ${ }^{34,37}$. Biomarkers that track astrocyte biology, such as GFAP, have been investigated over the past few years in neurodegenerative diseases. In AD, previous studies have shown that reactive astrocytes are closely associated with senile plaques and neurofibrillary tangles ${ }^{37-39}$. Elevated concentrations of GFAP in CSF and blood have been observed in AD, and it has been reported that its levels correlate with disease severity ${ }^{8-16,18}$. Recent studies have shown that elevated CSF and pGFAP concentration were associated with amyloid pathology, also in cognitively unimpaired subjects ${ }^{15-17,19}$. In addition, astrogliosis is also recognized in FTD. Astrogliosis is mainly confined to concrete regions such as frontal cortex and the hippocampus ${ }^{40,41}$. Interestingly, increased CSF and plasma GFAP concentrations have been reported both in sporadic and genetic FTD cohorts ${ }^{10-12,14,22}$. These findings suggest that PGFAP may be a valuable tool in FTD. According to this hypothesis, various studies observed elevated pGFAP concentration in different FTD subgroups and both in presenile and late-onset bvFTD cases ${ }^{23,24,26}$. In addition, one study in genetic FTDs found increased pGFAP concentration in progranulin-associated symptomatic FTD patients ${ }^{22}$. In agreement with previous studies, here we report an increased pGFAP concentration in FTD patients but to a lesser degree than that of AD. This result supports previous clinical-pathologic findings that show in FTD, there is less astrocytic activation and it is more confined to specific brain regions than in $A D 40$, 
41. On the other hand, the increase in $\mathrm{pNfL}$, a marker of axonal damage, suggests a similar degree of neurodegeneration in FTD compared to AD. One possible explanation for the differences found in pGFAP levels but not in pNfL is that FTD and AD are characterized by different neuroinflammatory patterns, with different degree of astroglia activation in different subpopulations but leading to a similar degree of neuronal degeneration. Similar to a prior study ${ }^{24}$, we found that pGFAP concentration tended to be different across the subtypes of the FTD spectrum, being significantly higher in svFTD and PSP-CBD compared to CN. This finding could indicate differences in the degree of astrocytic degeneration in different clinical phenotypes. ${ }^{22}$.

The current study showed that pGFAP had an acceptable performance to discriminate FTD from $A D(A U C=0.7)$ and $C N(A U C=0.76)$. These accuracies were somewhat lower than the values reported in two recent studies using pGFAP to identify bvFTD from AD (AUC $=0.85)$ and frontotemporal lobar degeneration (FTLD) from primary psychiatric disorders (AUC $=0.82)^{13,26}$. These discrepancies could be attributed to differences in cohort characteristics and heterogeneity due to different composition of FTD subtypes. An interesting finding was that, contrary to pNfL, which tend to be higher in FTD compared to AD, pGFAP levels were significantly lower in FTD, which suggests the potential diagnostic utility of their combination. Indeed, the combination of pGFAP with pNfL improved the diagnostic performance to distinguish FTD from AD and CN. Moreover, the incorporation of age, sex and APOE\& 4 allele improved the diagnostic performance up to an AUC of 0.83 in differentiating FTD from AD and AUC of 0.89 in differentiating FTD from CN. In addition, pGFAP was particularly promising in identifying AD, in agreement with previous observations ${ }^{13}$, 15, 17-19. Taken together, this study suggests that the combination of plasma astrocytic and neuronal markers could be relevant in FTD.

We observed that pGFAP concentration increased with age in controls, similarly to what has been observed in previous studies ${ }^{13,17,22,24}$, and we took age into account when interpreting this marker. In agreement with various recent reports ${ }^{15,17,20,22}$, pGFAP significantly correlated with CSF and plasma NfL, suggesting that glial activation and neuroaxonal degeneration are correlated. Previous studies have reported a weak correlation between CSF GFAP and YKL-40 levels in neurodegenerative diseases ${ }^{11,12}$. Similarly, we did not find significant correlation between pGFAP and CSF YKL-40 levels. This finding supports the hypothesis that different astrocyte subpopulations or different spatial distribution are involved in the pathophysiology of FTD and $A D^{12,37-41}$. The association between elevated pGFAP concentration, disease severity and prognostic markers in $A D$ has been reported before ${ }^{15,17-21}$. Previous studies reported that serum GFAP had potential to predict future conversion to dementia in cognitively normal individuals, not only to AD, but also to other dementias including FTD ${ }^{16,17,21}$. However, pGFAP as a marker of disease severity and prognosis in FTD has seldom been investigated ${ }^{13,24,25}$. In the current study, after adjusting by age and sex, pGFAP remained significantly correlated with cognitive and imaging measures in FTD. Higher concentration of pGFAP have been shown to be associated with lower brain volumes in GRN and C9orf72 presymptomatic carriers ${ }^{22}$, and its association with smaller hippocampal volume in SVPPA ${ }^{25}$ and temporal atrophy in FTLD has been reported ${ }^{26}$. To our knowledge this is the first study to show that higher concentration of pGFAP are also associated with orbitofrontal cortical thickness in the whole FTD group. In line with other reports in AD patients ${ }^{16,19,21}$, we observed that PGFAP predicted faster cognitive decline both in FTD and AD.

Association between pGFAP with cognitive decline in FTD had not been previously studied; our study shows a higher rate of cognitive decline among those with pGFAP $>240 \mathrm{pg} / \mathrm{ml}$. Surprisingly, the relationship between pGFAP and disease severity in AD was not confirmed in our cohort. One of the considerations to take into account when comparing our study with the previous ones is that others included both $A \beta+$ and $A \beta$ - subjects ${ }^{15,21}$, whereas in our study the correlation analysis was performed in disease subgroups. Previous studies found that pGFAP were increased in early stages of $A D^{15-21}$ and did not differ between disease stage ${ }^{18-19}$, which could explain the lack of association with imaging measures in the $A D$ subgroup. In agreement with previous studies $2,7,16,32$, our results show that glial biomarkers increase also in FTD besides AD, and its levels increase later in the disease course in FTD than in $A D$, when the disease is more advanced.

The strength of our study is the relatively large sample size, the inclusion of several FTD subgroups, regular follow-up of the subjects and multimodal approach, with inclusion of clinical measures, plasma, CSF and structural imaging. Additionally, all blood-based biomarkers were collected using the same standard operating procedures and measured following a harmonized protocol. This study has also some limitations. This study did not include serial longitudinal measures of pGFAP, the study lacks neuropathological confirmation, and we excluded AD copathologies in FTD groups.

\section{Conclusion}

In conclusion, our study shows that pGFAP concentration may be useful in FTD and could improve its diagnostic performance. Furthermore, our data support that pGFAP is not an exclusive marker in AD, but also play an essential role in other amyloid-independent processes involved in FTD. pGFAP could be useful as disease severity and prognostic marker in FTD.

\section{Abbreviations}

AD: Alzheimer's disease; AUC: Area under the curve; bvFTD: behavioral variant of Frontotemporal dementia; CN: cognitively normal participants; CSF: Cerebrospinal fluid; FTD: frontotemporal dementia; FTLD: frontotemporal lobar degeneration; FTD-FRS: Frontotemporal Dementia Rating Scale; GDS: Global Deterioration Scale of Reisberg; GFAP: Glial fibrillary acidic protein; HR: Hazard ratio; MMSE: Mini-Mental State Examination; NfL: neurofilament-light; nfvPPA: nonfluent variant of primary progressive aphasia; PET: Positron emission tomography; pGFAP: plasma Glial fibrillary acidic protein; pNfL: plasma neurofilament-light; PSP-CBD: progressive supranuclear palsy-corticobasal syndrome spectrum; ROC: receiver operating 
characteristic; SD: standard deviation; SIMOA: single molecule array; SPIN: Sant Pau Initiative on Neurodegeneration; svPPA: semantic variant of primary progressive aphasia.

\section{Declarations}

\section{Acknowledgements}

We are grateful to all participants in the study and their families. We also thank all the clinical team members that were involved in the selection and assessment of participants in the SPIN cohort, and the laboratory and neuroimaging teams for sample handling, biomarker analyses, and structural support.

\section{Ethics approval and consent to participate}

All procedures in the study were approved by the ethics committee at Hospital Sant Pau. All participants provided written informed consent.

\section{Consent for publication}

Not applicable.

\section{Availability of data and materials}

The datasets used and/or analyses during the current study are available from the corresponding author on reasonable request.

\section{Funding}

This study was supported by the Fondo de Investigaciones Sanitario (FIS), Instituto de Salud Carlos III (PI14/01126, PI17/01019 and PI20/01473 to JF, PI13/01532 and PI16/01825 to RB, PI18/00335 to MCI, PI18/00435 and INT19/00016 to DA, PI17/01896 and AC19/00103to AL) and the CIBERNED program (Program 1, Alzheimer Disease to AL), jointly funded by Fondo Europeo de Desarrollo Regional, Unión Europea, "Una manera de hacer Europa".

This work was also supported by Generalitat de Catalunya (2017-SGR-547, SLT006/17/125 to DA, SLT006/17/119 to JF, SLT002/16/408 to AL) and "Marató TV3" foundation grants 20141210 to JF, 044412 to RB and 20142610 to AL. This work was also supported by a grant from the Fundació Bancaria La Caixa to RB (DABNI project).

The sponsors of the study did not take part in the design and conduct of the study; collection, management, analysis and interpretation of the data; writing and review of the report; or the decision to submit the article for publication.

\section{Authors' contributions}

NZ, DA and AL designed the study. NZ, MS, IIG, VM, TE, IB, MA, JA, LM, OB, IS, MBSS, AS, LV, RB, JC, MCI, JF, AL and DA acquired data relevant for the study. NZ, DA and VM performed statical analysis. NZ, DA and AL contributed in analysis and interpretation of data. DA and AL participated in study supervision or coordination. NZ, DA, AL drafted the first version of the manuscript. All authors revised the manuscript for content and provided critical feedback.

\section{Competing interest}

Nuole Zhu is employed by Hospital de la Santa Creu i Sant Pau. Declarations of interest: none

Miguel Santos is employed by Hospital de la Santa Creu i Sant Pau. He is funded by a "Juan Rodés" research grant from the Institute of Health Carlos III.

Ignasio Illán-Gala is supported by the Global Brain Health Institute (Atlantic Fellow for Equity in Brain Health and pilot award for global brain health leaders CBHI ALZ UK-21-720973) and the “Juan Rodés” grant from the Institute of Health Carlos III /JR20/00018)

Victor Montal is employed by the Biomedical Research Institute Sant Pau. Declarations of interest: none

Teresa Estellés is employed by Biomedical Research Institute Sant Pau. Declarations of interest: Dr. Estellés is funded by a "Río Hortega" research grant from the Institute of Health Carlos III

Isabel Barroeta is employed by Hospital de la Santa Creu i Sant Pau. Declarations of interest: none

Miren Altuna is employed by Biomedical Research Institute Sant Pau. Declarations of interest: Dr. Estellés is funded by a "Río Hortega" research grant from the Institute of Health Carlos III 
Javier Arranz is employed by Biomedical Research Institute Sant Pau. Declarations of interest: none

Laia Muñoz is employed by Biomedical Research Institute Sant Pau. Declarations of interest: none

Olivia Belbin is employed by Biomedical Research Institute Sant Pau. Dr. Belbin is funded by a "Miguel Servet" research grant from the Institute of Health Carlos III

Isabel Sala is employed by Hospital de la Santa Creu i Sant Pau. Declarations of interest: none

M. Belén Sánchez-Saudinós is employed by the Biomedical Research Institute Sant Pau. Declarations of interest: none

Andrea Subirana is employed by the Biomedical Research Institute Sant Pau. Declarations of interest: none

Laura Videla is employed by Fundació Catalana Síndrome de Down. Declarations of interest: none

Rafael Bless is employed by Hospital de la Santa Creu i Sant Pau and received research grants from Institute of Health Carlos III, Fundació Bancària Obra Social La Caixa and Fundació La Marató de TV3. He participated in advisory boards from Lilly and Nutricia, and he received speakers honoraria and travel funding from Novartis and Nutricia

Jordi Clarimón is employed by Biomedical Research Institute Sant Pau and received research grants from Generalitat de Catalunya and from Institute of Health Carlos III

Maria Carmona-Iragu is employed by Hospital de la Santa Creu i Sant Pau. Declarations of interest: none

Juan Fortea is employed by Hospital de la Santa Creu i Sant Pau and received research grants from Institute of Health Carlos III, Fundació La Marató de TV3, and Pla Estratègic de Recerca i Innovació en Salut (PERIS)

Alberto LLeó is employed by Hospital de la Santa Creu i Sant Pau and received research grants from CIBERNED, Institute of Health Carlos III and Fundació BBVA. He participated in advisory boards from Fujirebio-Europe, Nutricia, Biogen and received speaker honoraria from Lilly

Daniel Alcolea is employed by Hospital de la Santa Creu i Sant Pau and received research grants from Pla Estratègic de Recerca i Innovació en Salut (PERIS SLT006/17/125), and from Institute of Health Carlos III (PI18/00435 and INT19/00016). He participated in advisory boards from FujirebioEurope and Roche Diagnostics and received speaker honoraria from Fujirebio-Europe, Roche Diagnostic, Nutricia, Esteve and from Krka Farmacéutica S.L.

\section{References}

1. Rohrer JD, Woollacott IO, Dick KM, Brotherhood E, Gordon E, Fellows A, Toombs J, Druyeh R, Cardoso MJ, Ourselin S, Nicholas JM, Norgren N, Mead S, Andreasson U, Blennow K, Schott JM, Fox NC, Warren JD, Zetterberg H. Serum neurofilament light chain protein is a measure of disease intensity in frontotemporal dementia. Neurology. 2016 Sep 27;87(13):1329-36.

2. Alcolea D, Vilaplana E, Suárez-Calvet M, Illán-Gala I, Blesa R, Clarimón J, Lladó A, Sánchez-Valle R, Molinuevo JL, García-Ribas G, Compta Y, Martí MJ, Piñol-Ripoll G, Amer-Ferrer G, Noguera A, García-Martín A, Fortea J, Lleó A. CSF sAPPß, YKL-40, and neurofilament light in frontotemporal lobar degeneration. Neurology. 2017 Jul 11;89(2):178-188.

3. Meeter LHH, Vijverberg EG, Del Campo M, Rozemuller AJM, Donker Kaat L, de Jong FJ, van der Flier WM, Teunissen CE, van Swieten JC, Pijnenburg YAL. Clinical value of neurofilament and phospho-tau/tau ratio in the frontotemporal dementia spectrum. Neurology. 2018 Apr 3;90(14):e1231-e1239.

4. Bridel C, van Wieringen WN, Zetterberg H, Tijms BM, Teunissen CE; and the NFL Group, Alvarez-Cermeño JC, Andreasson U, Axelsson M, Bäckström DC, Bartos A, Bjerke M, Blennow K, Boxer A, Brundin L, Burman J, Christensen T, Fialová L, Forsgren L, Frederiksen JL, Gisslén M, Gray E, Gunnarsson M, Hall S, Hansson O, Herbert MK, Jakobsson J, Jessen-Krut J, Janelidze S, Johannsson G, Jonsson M, Kappos L, Khademi M, Khalil M, Kuhle J, Landén M, Leinonen V, Logroscino G, Lu CH, Lycke J, Magdalinou NK, Malaspina A, Mattsson N, Meeter LH, Mehta SR, Modvig S, Olsson T, Paterson RW, Pérez-Santiago J, Piehl F, Pijnenburg YAL, Pyykkö OT, Ragnarsson O, Rojas JC, Romme Christensen J, Sandberg L, Scherling CS, Schott JM, Sellebjerg FT, Simone IL, Skillbäck T, Stilund M, Sundström P, Svenningsson A, Tortelli R, Tortorella C, Trentini A, Troiano M, Turner MR, van Swieten JC, Vågberg M, Verbeek MM, Villar LM, Visser PJ, Wallin A, Weiss A, Wikkelsø C, Wild EJ. Diagnostic Value of Cerebrospinal Fluid Neurofilament Light Protein in Neurology: A Systematic Review and Meta-analysis. JAMA Neurol. 2019 Jun 17;76(9):103548.

5. Karantali E, Kazis D, Chatzikonstantinou S, Petridis F, Mavroudis I. The role of neurofilament light chain in frontotemporal dementia: a metaanalysis. Aging Clin Exp Res. 2020 Apr 18.

6. van der Ende EL, Meeter LH, Poos JM, Panman JL, Jiskoot LC, Dopper EGP, Papma JM, de Jong FJ, Verberk IMW, Teunissen C, Rizopoulos D, Heller C, Convery RS, Moore KM, Bocchetta M, Neason M, Cash DM, Borroni B, Galimberti D, Sanchez-Valle R, Laforce R Jr, Moreno F, Synofzik M, Graff C, Masellis M, Carmela Tartaglia M, Rowe JB, Vandenberghe R, Finger E, Tagliavini F, de Mendonça A, Santana I, Butler C, Ducharme S,

Page 9/15 
Gerhard A, Danek A, Levin J, Otto M, Frisoni GB, Cappa S, Pijnenburg YAL, Rohrer JD, van Swieten JC; Genetic Frontotemporal dementia Initiative (GENFI). Serum neurofilament light chain in genetic frontotemporal dementia: a longitudinal, multicentre cohort study. Lancet Neurol. 2019 Dec;18(12):1103-1111.

7. Alcolea D, Martínez-Lage P, Sánchez-Juan P, Olazarán J, Antúnez C, Izagirre A, Ecay-Torres M, Estanga A, Clerigué M, Guisasola MC, Sánchez Ruiz D, Marín Muñoz J, Calero M, Blesa R, Clarimón J, Carmona-Iragui M, Morenas-Rodríguez E, Rodríguez-Rodríguez E, Vázquez Higuera JL, Fortea J, Lleó A. Amyloid precursor protein metabolism and inflammation markers in preclinical Alzheimer disease. Neurology. 2015 Aug 18;85(7):626-33.

8. Fukuyama R, Izumoto T, Fushiki S. The cerebrospinal fluid level of glial fibrillary acidic protein is increased in cerebrospinal fluid from Alzheimer's disease patients and correlates with severity of dementia. Eur Neurol. 2001;46(1):35-8.

9. Jesse S, Steinacker P, Cepek L, von Arnim CA, Tumani H, Lehnert S, Kretzschmar HA, Baier M, Otto M. Glial fibrillary acidic protein and protein S100B: different concentration pattern of glial proteins in cerebrospinal fluid of patients with Alzheimer's disease and Creutzfeldt-Jakob disease. J Alzheimers Dis. 2009;17(3):541-51.

10. Ishiki A, Kamada M, Kawamura Y, Terao C, Shimoda F, Tomita N, Arai H, Furukawa K. Glial fibrillar acidic protein in the cerebrospinal fluid of Alzheimer's disease, dementia with Lewy bodies, and frontotemporal lobar degeneration. J Neurochem. 2016 Jan;136(2):258-61.

11. Abu-Rumeileh S, Steinacker P, Polischi B, Mammana A, Bartoletti-Stella A, Oeckl P, Baiardi S, Zenesini C, Huss A, Cortelli P, Capellari S, Otto M, Parchi P. CSF biomarkers of neuroinflammation in distinct forms and subtypes of neurodegenerative dementia. Alzheimers Res Ther. 2019 Dec $31 ; 12(1): 2$.

12. Oeckl P, Weydt P, Steinacker P, Anderl-Straub S, Nordin F, Volk AE, Diehl-Schmid J, Andersen PM, Kornhuber J, Danek A, Fassbender K, Fliessbach K; German Consortium for Frontotemporal Lobar Degeneration, Jahn H, Lauer M, Müller K, Knehr A, Prudlo J, Schneider A, Thal DR, YilmazerHanke D, Weishaupt JH, Ludolph AC, Otto M. Different neuroinflammatory profile in amyotrophic lateral sclerosis and frontotemporal dementia is linked to the clinical phase. J Neurol Neurosurg Psychiatry. 2019 Jan;90(1):4-10.

13. Oeckl P, Halbgebauer S, Anderl-Straub S, Steinacker P, Huss AM, Neugebauer H, von Arnim CAF, Diehl-Schmid J, Grimmer T, Kornhuber J, Lewczuk P, Danek A; Consortium for Frontotemporal Lobar Degeneration German, Ludolph AC, Otto M. Glial Fibrillary Acidic Protein in Serum is Increased in Alzheimer's Disease and Correlates with Cognitive Impairment. J Alzheimers Dis. 2019;67(2):481-488.

14. Elahi FM, Casaletto KB, La Joie R, Walters SM, Harvey D, Wolf A, Edwards L, Rivera-Contreras W, Karydas A, Cobigo Y, Rosen HJ, DeCarli C, Miller $\mathrm{BL}$, Rabinovici GD, Kramer JH. Plasma biomarkers of astrocytic and neuronal dysfunction in early-and late-onset Alzheimer's disease. Alzheimers Dement. 2020 Apr;16(4):681-695.

15. Verberk IMW, Thijssen E, Koelewijn J, Mauroo K, Vanbrabant J, de Wilde A, Zwan MD, Verfaillie SCJ, Ossenkoppele R, Barkhof F, van Berckel BNM, Scheltens P, van der Flier WM, Stoops E, Vanderstichele HM, Teunissen CE. Combination of plasma amyloid beta (1-42/1-40) and glial fibrillary acidic protein strongly associates with cerebral amyloid pathology. Alzheimers Res Ther. 2020 Sep 28;12(1):118.

16. Milà-Alomà M, Salvadó G, Gispert JD, Vilor-Tejedor N, Grau-Rivera O, Sala-Vila A, Sánchez-Benavides G, Arenaza-Urquijo EM, Crous-Bou M, González-de-Echávarri JM, Minguillon C, Fauria K, Simon M, Kollmorgen G, Zetterberg H, Blennow K, Suárez-Calvet M, Molinuevo JL; ALFA study. Amyloid beta, tau, synaptic, neurodegeneration, and glial biomarkers in the preclinical stage of the Alzheimer's continuum. Alzheimers Dement. 2020 Oct;16(10):1358-1371.

17. Chatterjee P, Pedrini S, Stoops E, Goozee K, Villemagne VL, Asih PR, Verberk IMW, Dave P, Taddei K, Sohrabi HR, Zetterberg H, Blennow K, Teunissen CE, Vanderstichele HM, Martins RN. Plasma glial fibrillary acidic protein is elevated in cognitively normal older adults at risk of Alzheimer's disease. Transl Psychiatry. 2021 Jan 11;11(1):27.

18. Cicognola C, Janelidze S, Hertze J, Zetterberg H, Blennow K, Mattsson-Carlgren N, Hansson O. Plasma glial fibrillary acidic protein detects Alzheimer pathology and predicts future conversion to Alzheimer dementia in patients with mild cognitive impairment. Alzheimers Res Ther. 2021 Mar 27;13(1):68.

19. Pereira JB, Janelidze S, Smith R, Mattsson-Carlgren N, Palmqvist S, Teunissen CE, Zetterberg H, Stomrud E, Ashton NJ, Blennow K, Hansson O. Plasma GFAP is an early marker of amyloid- $\beta$ but not tau pathology in Alzheimer's disease. Brain. 2021 Jul 14:awab223.

20. Verberk IM, Laarhuis MB, van den Bosch KA et al. Serum markers glial fibrillary acidic protein and neurofilament light for prognosis and monitoring in cognitively normal older people: a prospective memory clinic-based cohort study. Lancet Healthy Long. 2021; 179:1373-84.

21. Rajan KB, Aggarwal NT, McAninch EA, Weuve J, Barnes LL, Wilson RS, DeCarli C, Evans DA. Remote Blood Biomarkers of Longitudinal Cognitive Outcomes in a Population Study. Ann Neurol. 2020 Dec;88(6):1065-1076.

22. Heller C, Foiani MS, Moore K, Convery R, Bocchetta M, Neason M, Cash DM, Thomas D, Greaves CV, Woollacott IO, Shafei R, Van Swieten JC, Moreno F, Sanchez-Valle R, Borroni B, Laforce R Jr, Masellis M, Tartaglia MC, Graff C, Galimberti D, Rowe JB, Finger E, Synofzik M, Vandenberghe R, de Mendonca A, Tagliavini F, Santana I, Ducharme S, Butler CR, Gerhard A, Levin J, Danek A, Frisoni G, Sorbi S, Otto M, Heslegrave AJ, Zetterberg H, Rohrer JD; GENFI. Plasma glial fibrillary acidic protein is raised in progranulin-associated frontotemporal dementia. J Neurol Neurosurg Psychiatry. 2020 Mar;91(3):263-270.

23. Marelli C, Hourregue C, Gutierrez LA, Paquet C, Menjot de Champfleur N, De Verbizier D, Jacob M, Dubois J, Maleska AM, Hirtz C, Navucet S, Bennys K, Dumurgier J, Cognat E, Berr C, Magnin E, Lehmann S, Gabelle A. Cerebrospinal Fluid and Plasma Biomarkers do not Differ in the Presenile and Late-Onset Behavioral Variants of Frontotemporal Dementia. J Alzheimers Dis. 2020;74(3):903-911.

Page $10 / 15$ 
24. Benussi A, Ashton NJ, Karikari TK, Gazzina S, Premi E, Benussi L, Ghidoni R, Rodriguez JL, Emeršič A, Binetti G, Fostinelli S, Giunta M, Gasparotti R, Zetterberg H, Blennow K, Borroni B. Serum Glial Fibrillary Acidic Protein (GFAP) Is a Marker of Disease Severity in Frontotemporal Lobar Degeneration. J Alzheimers Dis. 2020;77(3):1129-1141.

25. Heller C, Chan E, Foiani MS, Todd E, Russell LL, Greaves CV, Heslegrave AJ, Warren JD, Zetterberg H, Bocchetta M, Rohrer JD. Plasma glial fibrillary acidic protein and neurofilament light chain are measures of disease severity in semantic variant primary progressive aphasia. $\mathrm{J}$ Neurol Neurosurg Psychiatry. 2020 Nov 20:jnnp-2020-325085.

26. Katisko K, Cajanus A, Huber N, Jääskeläinen O, Kokkola T, Kärkkäinen V, Rostalski H, Hartikainen P, Koivisto AM, Hannonen S, Lehtola JM, Korhonen VE, Helisalmi S, Koivumaa-Honkanen H, Herukka SK, Remes AM, Solje E, Haapasalo A. GFAP as a biomarker in frontotemporal dementia and primary psychiatric disorders: diagnostic and prognostic performance. J Neurol Neurosurg Psychiatry. 2021 Jun 29:jnnp-2021326487.

27. Alcolea D, Clarimón J, Carmona-Iragui M, Illán-Gala I, Morenas-Rodríguez E, Barroeta I, Ribosa-Nogué R, Sala I, Sánchez-Saudinós MB, Videla L, Subirana A, Benejam B, Valldeneu S, Fernández S, Estellés T, Altuna M, Santos-Santos M, García-Losada L, Bejanin A, Pegueroles J, Montal V, Vilaplana E, Belbin O, Dols-Icardo O, Sirisi S, Querol-Vilaseca M, Cervera-Carles L, Muñoz L, Núñez R, Torres S, Camacho MV, Carrió I, Giménez S, Delaby C, Rojas-Garcia R, Turon-Sans J, Pagonabarraga J, Jiménez A, Blesa R, Fortea J, Lleó A. The Sant Pau Initiative on Neurodegeneration (SPIN) cohort: A data set for biomarker discovery and validation in neurodegenerative disorders. Alzheimers Dement (N Y). 2019 Oct 14;5:597609.

28. Rascovsky K, Hodges JR, Knopman D, Mendez MF, Kramer JH, Neuhaus J, van Swieten JC, Seelaar H, Dopper EG, Onyike CU, Hillis AE, Josephs KA, Boeve BF, Kertesz A, Seeley WW, Rankin KP, Johnson JK, Gorno-Tempini ML, Rosen H, Prioleau-Latham CE, Lee A, Kipps CM, Lillo P, Piguet O, Rohrer JD, Rossor MN, Warren JD, Fox NC, Galasko D, Salmon DP, Black SE, Mesulam M, Weintraub S, Dickerson BC, Diehl-Schmid J, Pasquier F, Deramecourt V, Lebert F, Pijnenburg Y, Chow TW, Manes F, Grafman J, Cappa SF, Freedman M, Grossman M, Miller BL. Sensitivity of revised diagnostic criteria for the behavioural variant of frontotemporal dementia. Brain. 2011 Sep;134(Pt 9):2456-77.

29. Gorno-Tempini ML, Hillis AE, Weintraub S, Kertesz A, Mendez M, Cappa SF, Ogar JM, Rohrer JD, Black S, Boeve BF, Manes F, Dronkers NF, Vandenberghe R, Rascovsky K, Patterson K, Miller BL, Knopman DS, Hodges JR, Mesulam MM, Grossman M. Classification of primary progressive aphasia and its variants. Neurology. 2011 Mar 15;76(11):1006-14.

30. Armstrong MJ, Litvan I, Lang AE, Bak TH, Bhatia KP, Borroni B, Boxer AL, Dickson DW, Grossman M, Hallett M, Josephs KA, Kertesz A, Lee SE, Miller BL, Reich SG, Riley DE, Tolosa E, Tröster Al, Vidailhet M, Weiner WJ. Criteria for the diagnosis of corticobasal degeneration. Neurology. 2013 Jan 29;80(5):496-503.

31. Höglinger GU, Respondek G, Stamelou M, Kurz C, Josephs KA, Lang AE, Mollenhauer B, Müller U, Nilsson C, Whitwell JL, Arzberger T, Englund E, Gelpi E, Giese A, Irwin DJ, Meissner WG, Pantelyat A, Rajput A, van Swieten JC, Troakes C, Antonini A, Bhatia KP, Bordelon Y, Compta Y, Corvol JC, Colosimo C, Dickson DW, Dodel R, Ferguson L, Grossman M, Kassubek J, Krismer F, Levin J, Lorenzl S, Morris HR, Nestor P, Oertel WH, Poewe W, Rabinovici G, Rowe JB, Schellenberg GD, Seppi K, van Eimeren T, Wenning GK, Boxer AL, Golbe LI, Litvan I; Movement Disorder Society-endorsed PSP Study Group. Clinical diagnosis of progressive supranuclear palsy: The movement disorder society criteria. Mov Disord. 2017 Jun;32(6):853-864.

32. Alcolea D, Martínez-Lage P, Sánchez-Juan P, Olazarán J, Antúnez C, Izagirre A, Ecay-Torres M, Estanga A, Clerigué M, Guisasola MC, Sánchez Ruiz D, Marín Muñoz J, Calero M, Blesa R, Clarimón J, Carmona-Iragui M, Morenas-Rodríguez E, Rodríguez-Rodríguez E, Vázquez Higuera JL, Fortea J, Lleó A. Amyloid precursor protein metabolism and inflammation markers in preclinical Alzheimer disease. Neurology. 2015 Aug 18;85(7):626-33.

33. Alcolea D, Pegueroles J, Muñoz L, Camacho V, López-Mora D, Fernández-León A, Le Bastard N, Huyck E, Nadal A, Olmedo V, Sampedro F, Montal V, Vilaplana E, Clarimón J, Blesa R, Fortea J, Lleó A. Agreement of amyloid PET and CSF biomarkers for Alzheimer's disease on Lumipulse. Ann Clin Transl Neurol. 2019 Sep;6(9):1815-1824.

34. Kwon HS, Koh SH. Neuroinflammation in neurodegenerative disorders: the roles of microglia and astrocytes. Transl Neurodegener. 2020 Nov 26;9(1):42.

35. Fischl B, Dale AM. Measuring the thickness of the human cerebral cortex from magnetic resonance images. Proc Natl Acad Sci U S A. 2000 Sep 26;97(20):11050-5.

36. Liddelow SA, Barres BA. Reactive Astrocytes: Production, Function, and Therapeutic Potential. Immunity. 2017 Jun 20;46(6):957-967.

37. Beach TG, Walker R, McGeer EG. Patterns of gliosis in Alzheimer's disease and aging cerebrum. Glia. 1989;2(6):420-36.

38. Delacourte A. General and dramatic glial reaction in Alzheimer brains. Neurology. 1990 Jan;40(1):33-7.

39. Ross GW, O'Callaghan JP, Sharp DS, Petrovitch H, Miller DB, Abbott RD, Nelson J, Launer LJ, Foley DJ, Burchfiel CM, Hardman J, White LR. Quantification of regional glial fibrillary acidic protein levels in Alzheimer's disease. Acta Neurol Scand. 2003 May;107(5):318-23.

40. Englund E, Brun A. Frontal lobe degeneration of non-Alzheimer type. IV. White matter changes. Arch Gerontol Geriatr. 1987 Sep;6(3):235-43.

41. Arnold SE, Han LY, Clark CM, Grossman M, Trojanowski JQ. Quantitative neurohistological features of frontotemporal degeneration. Neurobiol Aging. 2000 Nov-Dec;21(6):913-9.

\section{Figures}


A

pGFAP levels in FTD, AD and Control groups

C

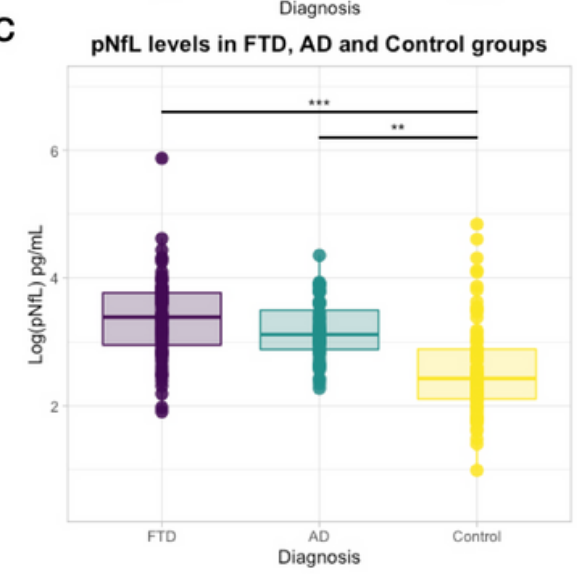

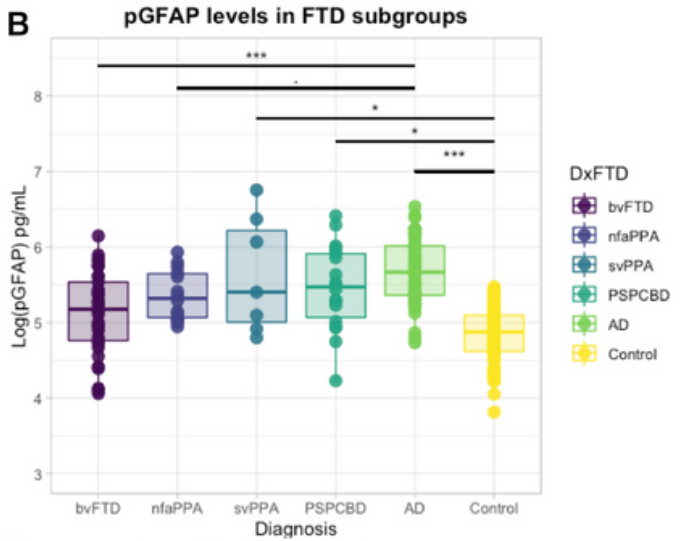

D pNfL levels in FTD subgroups

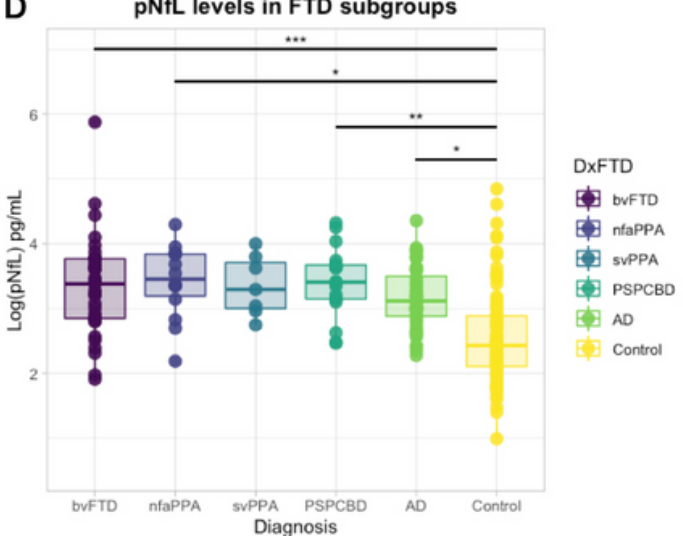

Figure 1

Comparison of pGFAP and pNfL in different groups: pGFAP in the FTD, AD and cog-nitively normal groups (A); pGFAP concentration in all FTD subgroups, $A D$ and cogni-tively normal groups (B); pNfL concentration in the FTD, AD and cognitively normal groups (C). pNfL concentration in all FTD subgroups, AD and cognitively normal groups (D). ${ }^{*} p<0.05,{ }^{*} \mathrm{p}<0.01,{ }^{* \star} \mathrm{p}<0.001$. Abbreviations: pGFAP = plasma Glial fibrillary acidic protein; pNfL = plasma Neurofilament light; $C N$ = cognitivelly nor-mal participants; FTD = Frontotemporal dementia; $A D$ = Alzheimer Disease
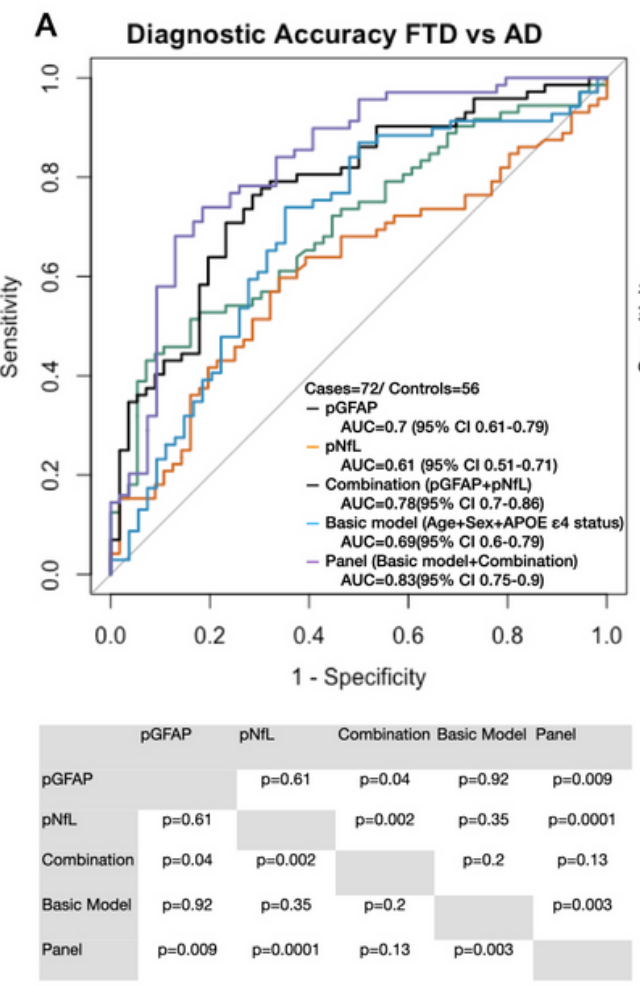
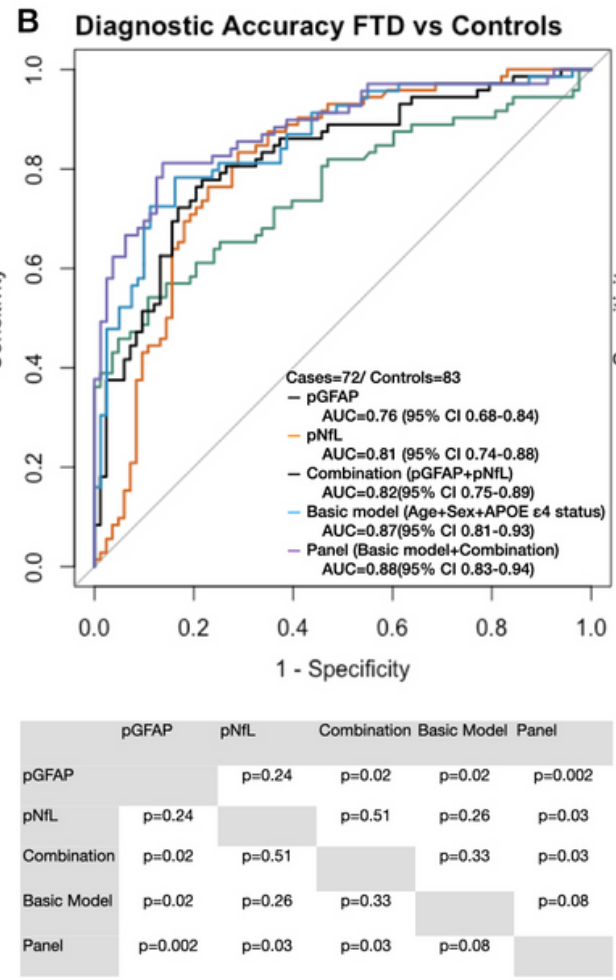

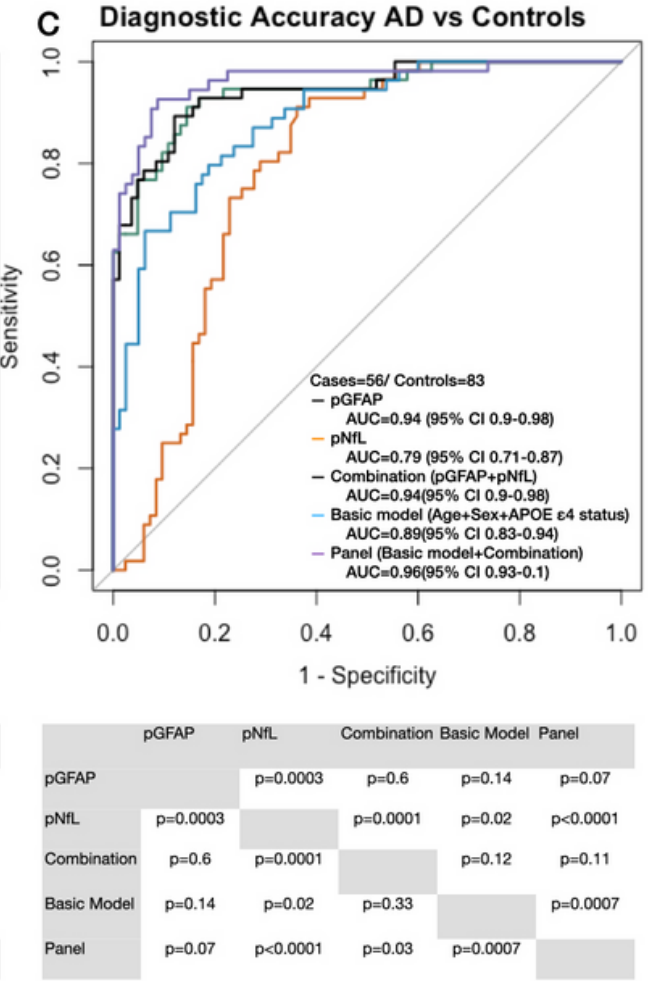




\section{Figure 2}

Receiver operating characteristic curves (ROC) for plasma GFAP, plasma neurofil-ament light, their combination, a basic model with risk factors (age, sex and APOE) and a combination of plasma biomarkers with additional risk factors to assess the accuracy to discriminate FTD from AD (A), FTD from normal cognitively normal (B), and AD from cognitively normal (C). Abbreviations: $A U C=$ area under the curve; $p G F A P=$ plasma Glial fibrillary acidic protein; $\mathrm{pNfL}$ = plasma Neurofilament light; $\mathrm{CN}=$ cognitivelly normal participants; $F T D=$ Frontotemporal dementia; $\mathrm{AD}=\mathrm{Alz}$-heimer Disease
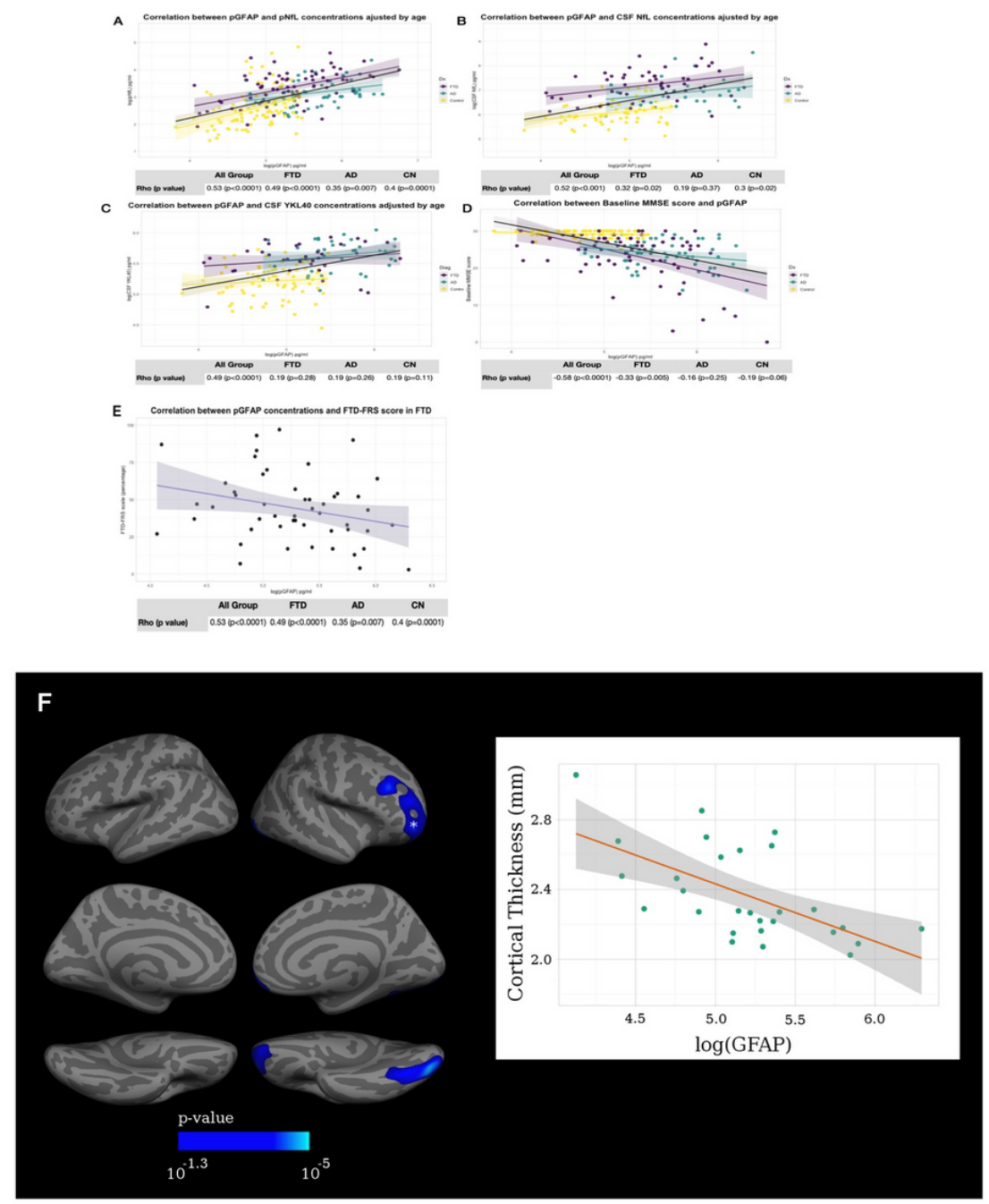

\section{Figure 3}

Correlation of pGFAP with plasma and CSF neurofilament light (A, B), CSF YLK (C), baseline MMSE score (D) in FTD, AD and normal cognitively. Correlation of plasma GFAP with baseline FTD-FRS score (E) and with cortical thickness in FTD ( $F, n=29)$. Blue regions represent a direct correlation. For illustrative purposes, ascatterplot shows the individual log (pGFAP) and the value of cortical thickness in the corre-sponding cortical region (marked with an asterisk) (F). P-value 10-1.3 $=0.05$. Al the correlation analysis are ajusted by age and sex. pGFAP = plasma Glial fibrillary acid-ic protein; $\mathrm{pNfL}$ = plasma Neurofilament light; $\mathrm{CN}=$ cognitivelly normal participants; FTD = Frontotemporal dementia; $\mathrm{AD}=\mathrm{Alzheimer}$ Disease 
A

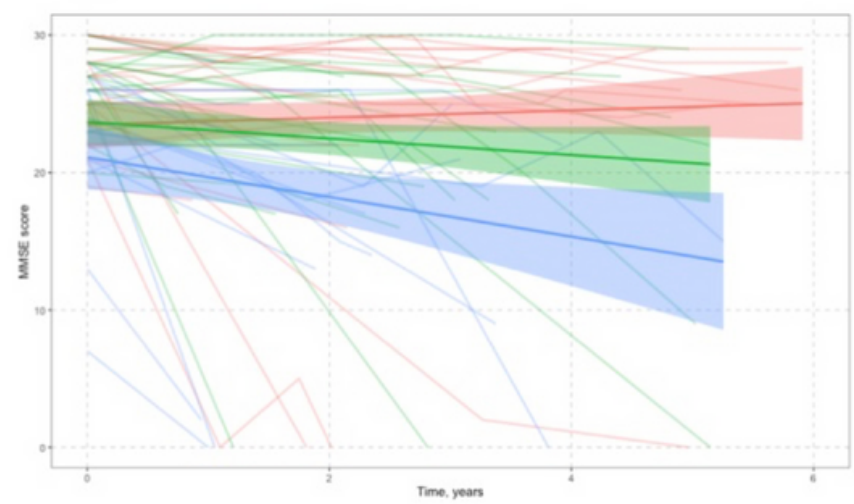

B

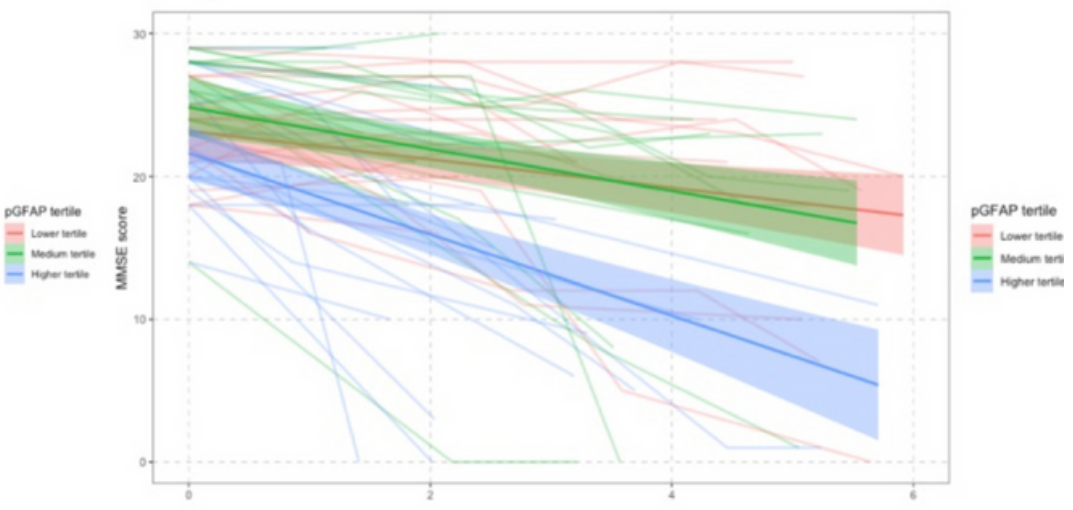

\section{Figure 4}

Relationship between baseline tertile of pGFAP and longitudinal changes of cogni-tive score (MMSE score) in FTD (A) and AD (B). Cognitive changes were estimated through linear mixed effects regression models adjusted for age and baseline MMSE score. Red lines show the lowest tertile (below $154 \mathrm{pg} / \mathrm{ml}$ in FTD and below 228pg/ml in AD), green lines show medium tertile (154-240pg/ml in FTD and 226-366pg/ml in AD), and blue lines show the highest tertile of biomarker concentra-tions (above $240 \mathrm{pg} / \mathrm{ml}$ in FTD and above $366 \mathrm{pg} / \mathrm{ml}$ in AD). Shaded areas indicate the $95 \%$ confidence for predicted cognitive scores. Abbreviations: $\mathrm{pGFAP}=$ plasma Glial fibrillary acidic protein; $\mathrm{pNfL}=$ plasma Neurofilament light; $\mathrm{CN}=$ cognitivelly normal participants; FTD = Frontotemporal dementia; $A D=$ Alzheimer Disease 

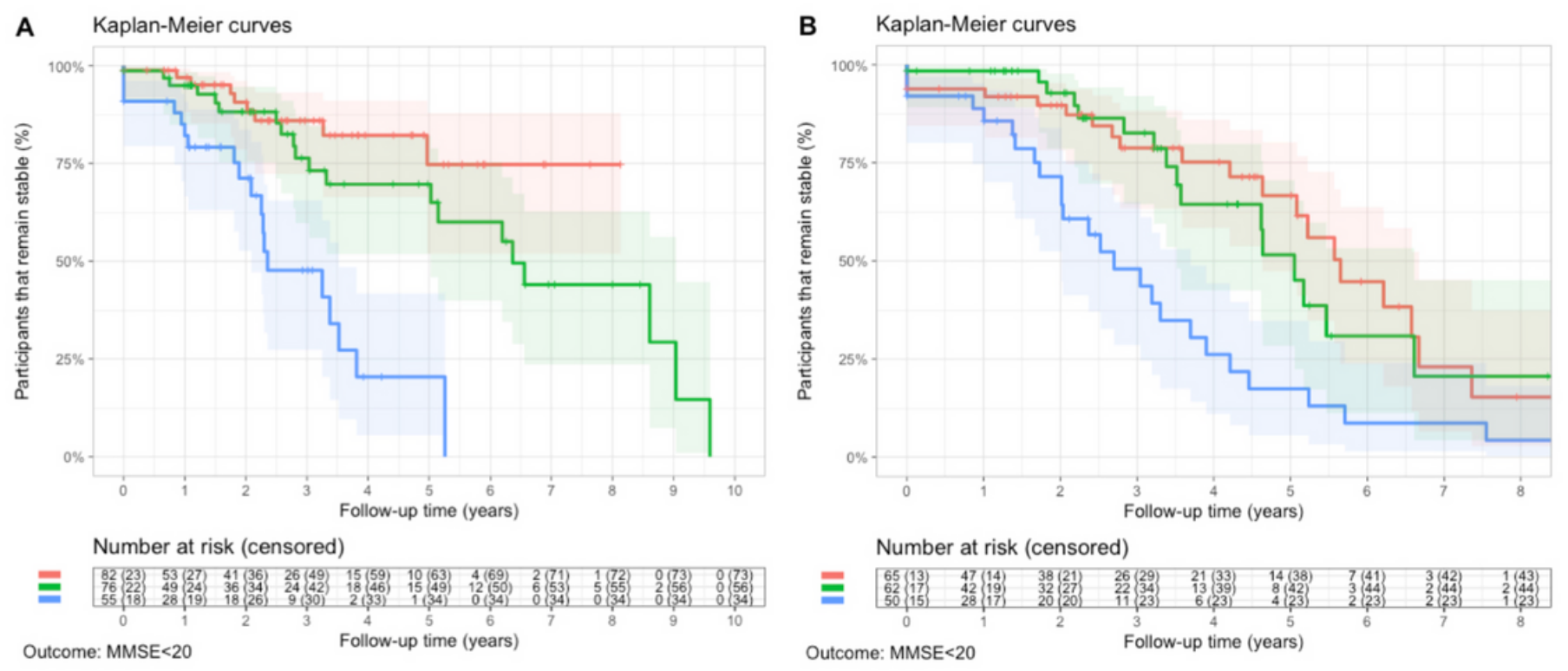

Profile - Lower tertile - Medium tertile - Higher tertile

\section{Figure 5}

Kaplan Meier curve of clinical progression to significant cognitive impairment (MMSE score<20) for individuals with low, medium or high baseline plasma GFAP in FTD (A) and in AD (B). Red lines show the lowest tertile (below 154pg/ml in FTD and below 228pg/ml in AD), green lines show medium tertile (154-240 pg/ml in FTD and 226-366pg/ml in AD), and blue lines show the highest tertile of biomarker concen-trations (above $240 \mathrm{pg} / \mathrm{ml}$ in FTD and above $366 \mathrm{pg} / \mathrm{ml}$ in AD). In FTD, the median time of progression to significant cognitive impairment is was 6.4 (5-9) years in medium tertile pGFAP group and 2.4 (2.1-3.5) years in high tertile pGFAP group (Log-rank test, $p<0.0001)$. In $A D$, the median time of progression to significant cog-nitive impairment is was 5.7 (4.6-6.7) years in low tertile pGFAP group, 5.1 (3.5-6.6) years in medium tertile pGFAP group and 2.7 (23.7) years in high pGFAP tertile group (Log-rank test, $\mathrm{p}=0.0002)$. Abbreviations: pGFAP = plasma Glial fibrillary acid-ic protein; FTD = Frontotemporal dementia; $A D=$ Alzheimer Disease 